\title{
The Art of a Complex Giant System: Supercomputer in the World and China
}

\author{
Si Hongwei \\ Department of the History of Science, Tsinghua University, Beijing, China
}

Email address:

howes@mail.tsinghua.edu.cn

\section{To cite this article:}

Si Hongwei. The Art of a Complex Giant System: Supercomputer in the World and China. History Research. Vol. 9, No. 1, 2021 , pp. 58-64. doi: $10.11648 /$ j.history.20210901.17

Received: March 1, 2021; Accepted: March 16, 2021; Published: March 26, 2021

\begin{abstract}
Supercomputer also known as gigantic computer, refers to a kind of computer with the best performance, the strongest function, the fastest operation speed and the largest storage capacity among all kinds of computers in an era. Supercomputer is a kind of complex electronic giant system, has huge numerical calculation ability and data processing ability, and can calculate large and complex tasks that ordinary personal computers and servers cannot complete. It has been used in high-end technology and cutting-edge scientific research at the beginning. With the continuous progress of science and technology, the upgrading speed of computer is very fast. Therefore, Supercomputer has distinct temper of times, and its evaluation standard is also dynamic. It is an important embodiment of scientific and technological strength of the country and plays a huge role in promoting national economic development, social progress and national defense construction. The paper reviews the development of the international supercomputer and overview of supercomputer development in China. It analyzes the current situation of supercomputer R\&D, shows the supercomputer technology problem and innovation-storage access wall, communication wall, reliability wall, energy consumption wall. This paper also looks forward to the future of supercomputer, so as to provide some reference for scholars in related fields.
\end{abstract}

Keywords: Giant System, Supercomputer, World, China

\section{Introduction}

Supercomputer, also known as gigantic computer, refers to a kind of computer with the best performance, the strongest function, the fastest operation speed and the largest storage capacity among all kinds of computers in an era and it is also a complex electronic giant system. [1] Supercomputer has huge numerical calculation ability and data processing ability, and can calculate large and complex tasks that ordinary personal computers and servers cannot complete. With the continuous progress of science and technology, the upgrading speed of computer is very fast. Therefore, Supercomputer has distinct temper of times, and its evaluation standard is also dynamic. For example, in the mid-1980s, it stipulated that a digital electronic computer with floating-point operation speed of more than 100 million times per second, word length of 64 bits and main memory capacity of $4-16 \mathrm{MB}$ was considered a supercomputer in the industry field. [2]

In 1946, ENIAC, the first electronic computer in the world, was created at the University of Pennsylvania in the United
States, which opened "computer and information age". With the improvement of semiconductor and integrated circuit technology, computers develop rapidly, resulting in the emergence of small, medium and large computers, which are widely used in industries, science and technology, universities, governments, military and other fields and departments all over the world, greatly promoting the development of productivity. However, with the progress of science and technology, many scientific computing problems, such as aerodynamics, nuclear physics, space technology, medium and long-term weather forecast, oil exploration, seismic data processing, cipher decoding, require calculation with a large amount of numbers, complexity and high accuracy; and human "big science" projects such as the "Apollo" moon landing program are large-scale, involving a wide range, which the general computer is difficult to complete. It is urgent to develop a computer system with faster processing speed, larger storage capacity and higher input / output transmission rate.

With the characteristics of high integration, small size, low 
power consumption and good reliability, the development of integrated circuits has laid a material foundation for the design and manufacture of computer systems with more complex structure and stronger processing ability. At the same time, great progress has been made in computer software, with the functions of operating system continuously enhanced and improved, various high-level languages and their compilers widely used, and various algorithms gradually improved. All of these provide technical support for the development and application of computer system software with better performance.

In the case of demand traction and technology promotion, supercomputer system came into being. It has been used in high-end technology and cutting-edge scientific research at the beginning. It is an important embodiment of scientific and technological strength of the country and plays a huge role in promoting national economic development, social progress and national defense construction.

\section{The Review of the Development of the International Supercomputer}

In the world, the research of Supercomputer first started in the United States. After the Second World War, in order to dominate the world, the United States actively expanded its military, vigorously developed strategic nuclear weapons with strong deterrent force, established and strengthened the National Laboratory for the research and development of military cutting-edge technology, and carried out in-depth research on the basic theory of atomic energy and thermonuclear weapons, all of which involve a large number of complex calculations. After the advent of ENIAC, the performance of computers has been continuously improved and upgraded from electron tube to transistor, but it still cannot meet the needs of the U.S. government and military. They put forward higher requirements for computer manufacturers in terms of computing speed, machine performance, stability and reliability. IBM, UNIVAC and $\mathrm{CDC}$, the main computer companies in the United States, participated in the research and development of the early Supercomputer system. [3]

In the 1950s, the Radiation Laboratory of the University of California, Livermore Laboratory, invited tenders for high-performance decimal computers. UNIVAC won the fierce competition with IBM, won the contract of Livermore to atomic research computer LARC, and delivered the machine 29 months later. Then, the Los Alamos Scientific Laboratory of the Atomic Energy Commission of the United States urgently needed a "supercomputer" that was two orders of magnitude better than the best computer at that time. IBM won the bid and began to develop a computer that was 100 times better than any existing computer in 1956, which was the famous IBM stretch (IBM7030) computer at that time. This super transistor electronic circuit computer named "extended" is the first major product of IBM company. It adopts many innovative technologies, including instruction pipeline technology, instruction pre-decoding, advanced control, memory operands prefetching, out of order execution, speculative execution based on transfer prediction, transfer prediction error recovery and precise interrupt, etc. these technical standards even are still used today. In 1960, Stretch was successfully developed, but it did not reach the original performance criteria, and its speed only reached about $60 \%$ of the expected speed. Although Stretch became the fastest computer in the world at that time, the performance gap made IBM feel very embarrassed, and stopped further production of the machine in May 1961.

The first person who developed a product that meets the definition of supercomputer is Seymour Cray (1925-1996), who is later known as "the father of Supercomputer". [4] Clay was born in Wisconsin. His father was a civil engineer. His father's dedication to technology and his pursuit of perfection have a subtle influence on clay's life. In 1960, the newly established 3-year-old Control Data Company (CDC) accepted the Commission of the US Atomic Energy Commission and began to develop Supercomputers. The chief computer designer of the company was Cray, who was only 31 years old. He led the team to launch the famous CDC6600 in August 1964. CDC6600 was the fastest large-scale scientific computer in the world at that time, with 1 million floating-point operations per second, about three times faster than IBM Stretch. It was the fastest computer in the world from 1964 to 1969, until its successor, CDC7600, was transferred.

Then in the 1970s, Cray, who left CDC and founded Cray Company, began to design and manufacture a supercomputer which give the first place on vector supercomputing. Cray-1 was born in 1975, creating a super speed of 100 million operations per second, which was unparalleled in the world at that time. It was strictly tested in Los Alamos National Laboratory for a long time, and was officially launched in 1976, which caused a sensation in the global computer and scientific circles. Cray-1 adopts a series of technological innovations, such as ECL high-speed integrated circuit, vector data type and vector computing, high-density assembly technology and high-efficiency cooling technology. It not only has high performance, but also has a maximum peak vector speed of 160 million floating-point operations per second. It has become a real supercomputer, highly praised and welcomed by the computer industry and supercomputer users, and has won the market of scientific computing and super large scale data processing. Moreover, the simplicity and novelty of its architecture has actually become the standard model of vector computers, so that the vector computers developed and produced in many countries are based on Cray-1. Later, Cray continued to design and manufacture Cray-2, Cray-3 and Cray-4, constantly climbing one peak after another in the development of supercomputer technology innovation. Since then, supercomputer has become the upstart of science and technology, widely used in nuclear energy research, aerospace, life science, oil exploration, earthquake prediction and other fields, Supercomputing began to become the third main way of scientific activities besides experiment and theory.

In 1993, Hans Meuer (1936-2014), Erich Strohmaier and 
other professors of Mannheim University in Germany initiated and create the global top 500 Supercomputer Ranking List. Later, they jointly held it with the United States and released the authoritative ranking list of world supercomputers, twice a year, which became one of the important standards to evaluate the supercomputer level all over the world.

When launching the TOP500 project, the founders put forward the guiding principles: first, ranking the 500 most powerful computers in the world; second, benchmarking with the best Linpack performance; third, updating and publishing the ranking list twice a year, ISC (International Supercomputing Conference) in Germany in June, SC (Supercomputing Conference) in the United States in November; fourth, All the data are in www.top500.org For public inspection.

Since the world's first supercomputer came to being in the United States, the technology and industry of supercomputer in the United States has been in the forefront of the world, occupying the first place in the top 500 list for most of the time before 2010, and gradually surpassed by China after 2010 . Over the past decades, the United States has developed most of the cutting-edge technologies for supercomputers, and has produced a number of supercomputers with the largest scale and fastest speed in many of its research laboratories and universities. Some of the top supercomputers are used to simulate nuclear tests, while others are used for research in weather forecasting, energy exploration and other fields.

In addition to the United States, Japan, Europe, Russia and India are also engaged in the development of supercomputers.

Japan is also one of the earliest countries that develop supercomputers. [5] In 1999, Japan invested heavily in the development of the supercomputer called "Earth Simulator" to simulate the earth's activities and engage in earth science. In 2002, as soon as the machine was launched, the United States, which ranked first in the top 500 list, was knocked off. Since then, due to funding reasons, the research and development of supercomputers in Japan has been led by the government to the direction of integration of production, teaching and research and providing more services for the public. However, in June 2011, the "K" supercomputer, which was developed by Fujitsu and assembled by the Japan Institute of Physics and Chemistry, and funded by the Japanese government, became the world's fastest supercomputer at 8162 trillion operations per second. Japan returned the first place in the supercomputer field in the world after seven years.

In Europe, supercomputers are developed jointly by many countries. In June 2010, the "Supercomputer Cooperation Platform Project" participated by European countries was launched in Barcelona, Spain. [5] The purpose of the project is to build a large-scale infrastructure connecting various computing resources in Europe, and enhance the supercomputing capacity of the whole Europe.

The fastest supercomputer in Russia is called Lomonosov, developed by Moscow University with a peak speed of 170 million times. India is also one of the few countries that own supercomputers in the world, developing high-quality and high-speed supercomputers at low cost. [5] At present, the Russian Academy of Sciences plans to cooperate with relevant supercomputer companies in India to build faster supercomputers.

Table 1. The world's top 500 Supercomputers over the years since TOP500 Ranking List was born.

\begin{tabular}{|c|c|c|c|}
\hline Name & Manufacturer & Country & Time be the top in the world \\
\hline CM-5 & TMC & America & June in 1993-Decmber in 1993 \\
\hline Numerical wind tunnel & Fujitsu & Japan & December in 1993-June in 1994 \\
\hline Paragon XP/S140 & Intel & America & June in 1994-Decmber in 1994 \\
\hline Numerical Wind Tunnel2 & Fujitsu & Japan & December in 1994-June in 1996 \\
\hline SR2201 & Hitachi & Japan & June in 1996-December in 1996 \\
\hline CP-PACS & Hitachi & Japan & December in 1996-June in 1997 \\
\hline ASCI Red & Intel & America & June in 1997-December in 2000 \\
\hline Earth simulator & Japanese electronics company & Japan & June in 2002-December in 2004 \\
\hline Blue gene / L & IBM & America & December in 2004-June in 2008 \\
\hline Roadrunner & IBM & America & June in 2008-December in 2009 \\
\hline Jaguar & Cray & America & December in 2009-December in 2010 \\
\hline Tianhe-1A & National University of Defense Technology & China & December in 2010-June in 2011 \\
\hline K & Fujitsu & Japan & June in 2011-June in 2012 \\
\hline Sequoia & IBM & America & June in 2012-December in 2012 \\
\hline Sunway TaihuLight & National Parallel Computer Engineering Technology Center & China & June in 2016-June in 2018 \\
\hline Vertex & IBM & America & June in 2018-June in 2020 \\
\hline Fugaku & Fujitsu & Japan & June in 2020- \\
\hline
\end{tabular}

\section{Overview of Supercomputer Development in China}

Computer research in China began in the 1950s. After the founding of the Chinese Academy of Sciences, Hua Luogeng was appointed director of the Institute of Mathematics. With his strong support, China's first computer research group was officially established in January 1953. In 1956, the Chinese Academy of Sciences set up the Preparatory Committee for the Institute of Computing Technology, and joined hands with 
a number of capable scientific and technological forces from the central, local and military units to jointly prepare for the establishment of the Institute of Computing Technology. In 1959, the Institute successfully developed the first large fast electronic computer for general purpose in China. [6-8]

In the 1960s, relying on its own strength, China successfully developed "two bombs and one satellite", greatly improving its national defense strength and international status. But at that time, China's cutting-edge science and technology also encountered obvious development bottlenecks. Some important theoretical research and simulation experiments, especially the development of strategic nuclear weapons, the research of nuclear power plants, the design of aerospace vehicles, military intelligence analysis and satellite image interpretation and etc. needed to solve a large number of computing problems before new breakthroughs could be made. Therefore, it is urgent to have extremely high computing speed Supercomputers. Medium and long-term numerical weather forecast, reservoir engineering and energy development, large-scale graphics and image processing, and biological genetic engineering are also needed. [9-10]

In March 1978, the National Science Conference was held in Beijing. Deng Xiaoping pointed out at the meeting: "if China wants to carry out the four modernizations, it cannot do without supercomputers." He entrusted the research and development task to National University of Defense Technology (NUDT). [11]

As the chief designer, Professor Ci Yungui (1917-1990) led the scientific research team to successfully develop China's first 100 million times supercomputer "YinHe"(YH-1) after five years of hard work, breaking the international high-tech blockade, making China the third country in the world after the United States and Japan that can independently develop supercomputers. Professor Ci Yungui is known as "the father of China's Supercomputer". In December 1983, "YH-1" successfully passed the national appraisal and reached the domestic advanced level. In some aspects, it has reached the international level. Its successful development has filled the gap in the supercomputers field in China, marked that China has entered the ranks of developing supercomputers in the world, and provided valuable experience for the development of China's computer work. [12-13]

Since then, the NUDT has successively developed China's first YH-series supercomputer "YH-2" and 10 billion supercomputer "YH-3" and others, which are widely used in numerical weather forecasting, nuclear energy development, aerospace, oil and seismic data processing and other fields, and have played an important role in China's national defense construction and national economic development. In November 2010, TH-1, China's first 10 billion times supercomputer developed by the unit, beat the United States in the top 500 list, won the championship and shocked the world. Since June 2013, TH-2, China's first 100 million times supercomputer, has been ranked the first place in the top 500 supercomputers list in the world with sixth straight title. As the business host of Guangzhou National Supercomputer Center, TH-2 has been applied in many fields, such as cloud computing and large data, and has been gradually applied in a series of fields, such as life science, geophysics, large-scale genome assembly, environmental pollution control, and begin to make "Chinese Supercomputing" famous all over the world. [14-15]

In addition to the development of the "YH" supercomputer by the NUDT, the National Parallel Computer Engineering Technology Center (NPCETC), the Institute of Computing Technology- Chinese Academy of Sciences (ICTCAS), and Lenovo Company are also engaged in supercomputer research, launching a number of China's home-made supercomputer systems such as "Sunway", "Sugon" and "ShenTeng". [16]

In 1996, NPCETC was established, which began the development of "Sunway" series supercomputer system. In 1999, Sunway-1 supercomputer was set up in the National Meteorological Administration of Beijing. The peak value of the system is 384 billion floating-point operations, and the application is good. In 2010, Sunway-BlueRay was born with a computing speed of 1100 trillion times per second. For the first time, it completely adopted China's own CPU. In June 2016, the "Sunway TaihuLight" supercomputer developed by the company reached the top 500, won three "world No.1" records of peak performance of 125.436pflops, sustained performance of $93.015 \mathrm{pflops}$ and performance power consumption ratio of $6051 \mathrm{mflops} / \mathrm{W}$, and was certified by Guinness World Records, becoming the world's first supercomputer system with computing speed over 1 billion times. In November 2016, "Sunway TaihuLight" won the first place in the world's supercomputer rankings. At the same time, the scientific research team of Tsinghua University, with the application of "global atmospheric non static cloud resolution simulation" running on "Sunway TaihuLight", won the highest international supercomputer application Award - "Gordon bell" award for the first time.

Table 2. Major events of supercomputer development in China.

\begin{tabular}{|c|c|c|c|}
\hline Time & Machine & $\begin{array}{l}\text { Development } \\
\text { department }\end{array}$ & Peak speed \\
\hline 1983 & YH-1 & NUDT & 100MFlops \\
\hline 1992 & YH-2 & NUDT & 1GFlops \\
\hline 1993 & Sugon-1 & ICTCAS & 0.64GFlops \\
\hline 1995 & Sugon-1000 & ICTCAS & 2.5GFlops \\
\hline 1996 & Sugon-2000 & ICTCAS & 4GFlops \\
\hline 1997 & YH-3 & NUDT & 13GFlops \\
\hline 1998 & Sugon-2000 I & ICTCAS & 20GFlops \\
\hline 1999 & Sugon-2000 II & ICTCAS & 111.7GFlops \\
\hline 1999 & Sunway-1 & NPCETC & 384GFlops \\
\hline 2000 & Sugon-3000 & ICTCAS & 403.2GFlops \\
\hline 2000 & YH-X & NUDT & 1TFlops \\
\hline 2002 & ShenTeng-1800 & Lenovo Group Ltd. & 1TFlops \\
\hline 2003 & Sugon-4000L & ICTCAS & 4.2TFlops \\
\hline 2003 & ShenTeng- 6800 & Lenovo Group Ltd. & 5.3TFlops \\
\hline 2004 & Sugon-4000A & ICTCAS & 11TFlops \\
\hline 2007 & Sunway-3000A & NPCETC & 18TFlops \\
\hline 2008 & ShenTeng-7000 & Lenovo Group Ltd. & 106.5TFlops \\
\hline 2008 & Sugon-5000A & ICTCAS & 230TFlops \\
\hline 2009 & TH-1 & NUDT & 1.206PFlops \\
\hline 2010 & Sunway-BlueRay & NPCETC & 1.1PFlops \\
\hline 2010 & Sugon-Nebula & ICTCAS & 1.271PFlops \\
\hline 2010 & TH-1A & NUDT & 4.7PFlops \\
\hline 2013-2015 & TH-2 & NUDT & 54.9PFlops \\
\hline 2016 & Sunway TaihuLight & NPCETC & 125.436PFlops \\
\hline
\end{tabular}


"Sugon" supercomputers are developed by Institute of Computer Science, Chinese Academy of Sciences. In October 1993, after the launch of China's first fully symmetric shared memory multiprocessing supercomputer "Sugon-1", the Institute immediately established Sugon computer company, and then produced more than 30 types of Sugon super servers based on technologies such as Sugon-2000, Sugon-3000, Sugon-4000 and Sugon-5000A. In June 2010, "Sugon-Nebula" supercomputer entered the second place in the world's supercomputer rankings, reaching the best performance of China's "Sugon" series supercomputers.

Lenovo Company is also one of the research and development forces of China's supercomputer system. In 2002, ShenTeng-1800, China's first enterprise developed trillion sub supercomputer, was born in Beijing. It ranked 43rd in the top 500 list in November of that year with the measured performance of 1.046 trillion floating-point operations per second. This is the first time that the supercomputer developed by China's enterprises has entered the top 500, marking that the "giant ship" of China's IT enterprises has entered the world's super supercomputer The "deep sea" of computer research and development.

\section{Current Situation of Supercomputer R \& D}

Supercomputer is an important means to solve a series of major problems involving a country's economy, society, science, security, military and other fields. It is an important symbol of comprehensive national strength and scientific and technological strength. It is an important component of the national innovation system. It has increasingly become a strategic commanding point for world powers.

At present, there are several characteristics in the world's supercomputer field:

1. The world powers are competing to keep the leading position in the development of supercomputers, and the competition in this field has become ultra-intense. After China's "Tianhe-1" won the first place in the world in 2010, it further intensified international competition. Japan invested more than US \$1 billion in " $K$ " supercomputer and won the first place in the world ahead of time in June 2011; the United States successively launched "Sequoia" of IBM and "Titan" of Cray, and won the first place in the world in 2012; China's "Tianhe-2" and "Sunway TaihuLight" won the top 500 world champion for ten times from 2013 to 2018; the United States "apex" supercomputer won the first place in the world again in June 2018; In June 2020, "Fugaku" of Japan will surpass "Summit" of the United States and become the world's fastest supercomputer.

2. Under the background of the international new coronavirus pandemic and the rising tide of antiglobalization, the West regards the development of supercomputers as a tool to revitalize the real economy and strengthen trade protectionism, which can not only directly drive the development of microelectronics and microprocessors, high-end software research and development, optical communication and other related industries, but also indirectly support financial big data analysis, biomedicine, new energy and new materials And other high-tech emerging industries. The United States is pushing the supercomputer technology to a stage of deepening the development of the whole industry chain integration. The era of supercomputer widely used by small and medium-sized enterprises is coming.

3. At present, the advantages of the United States and other powerful countries in the field of supercomputers are due to the strong natural science, long-term continuous huge investment and the breakthrough of core key technologies, as well as the overall leading position of its information industry. Although China's supercomputers have been leading the world several times, "Sunway TaihuLight" and "Tianhe-2" have occupied the highest position in the top 500 list for four consecutive years, and China's machine share on the list has also increased year by year, the leading advantage of the western power groups, especially the United States, has not changed. According to the latest world supercomputer ranking released in November 2020 [17], all the top ten countries are western countries except the fourth and sixth "Sunway TaihuLight" and "Tianhe-2" from China. Among them, the United States has four machines in the top ten. On the whole, the United States accounts for $33.9 \%$ of the total global supercomputer development capacity, ranking first. IBM of the United States is the largest manufacturer of supercomputer CPU in the world, and AMD of the United States ranks second. $96 \%$ of all supercomputers on the list use core accelerators equipped with NVIDIA GPU.

4. "Collaborative innovation" is becoming an important way to support the sustainable development of supercomputer technology. Supercomputer research and development is changing from the super large-scale single system research and development to the "whole ecosystem" development mode combining enabling technology, system technology and application technology. This development model makes full use of the collaborative innovation of companies, universities and research institutions, and continues to increase the collaborative innovation in microprocessor design, process structure, programming model and other fields. At the same time, the collaborative innovation in application field is deepening, the supercomputing application models in the United States and Japan are becoming more and more mature, and China has successively established the National Supercomputing Tianjin Center, Guangzhou center, Changsha center, Shenzhen Center, Jinan center, Wuxi center. It begins to develop higher performance supercomputers using collaborative innovation model, at the same time large-scale integrated circuit design center, basic software engineering center and industrialization base will be built. 
Table 3. The latest top 10 supercomputers in the world (by November 2020).

\begin{tabular}{|c|c|c|c|c|c|}
\hline Ranking & Name & Developer & Processor core & Installation site & Peak speed \\
\hline 1 & Fugaku & Fujitsu, Japan & 7630848 & advanced high performance computing Joint Center in Japan & $442010 \mathrm{TFlop} / \mathrm{s}$ \\
\hline 2 & Summit & IBM, USA & 2414592 & Department of energy Oak Ridge National Laboratory & $148600 \mathrm{TFlop} / \mathrm{s}$ \\
\hline 3 & Sierra & IBM, USA & 1572480 & Lawrence Livermore National Laboratory, U.S. Department of energy & $94640 \mathrm{TFlop} / \mathrm{s}$ \\
\hline 4 & $\begin{array}{l}\text { Sunway } \\
\text { TaihuLight }\end{array}$ & NPCETC, China & 10649600 & National Supercomputing Center, Wuxi, China & 93015 TFlop/s \\
\hline 5 & Selene & Cray, USA & 555520 & Swiss National Computing Center & 63460 TFlop/s \\
\hline 6 & Tianhe-2 & NUDT, China & 4981760 & National Supercomputer Center, Guangzhou, China & 61445 TFlop/s \\
\hline 8 & HPC5 & Petroleum, Italy & 669760 & Italian supercomputing & 35450 TFlop/s \\
\hline 9 & Frontera & Cray, USA & 448448 & Berkeley Laboratory, National Center for energy research and scientific computing & 23516 TFlop/s \\
\hline 10 & Dammam-7 & HPE, Saudi & 672520 & Aramco laboratory, Saudi Arabia & $22400 \mathrm{TFlop} / \mathrm{s}$ \\
\hline
\end{tabular}

\section{Future Prospects of Supercomputer Technology Innovation}

The history of supercomputer development since 1960s shows that the speed of supercomputer is about "1000 times higher than before every 10 years". [18] Supercomputer has achieved 10 billion times $\left(10^{\text {th }}\right.$ power $)$ in June 2020 , and the world has entered the era of E-level (Exascale) computing.

After all, supercomputer is not a simple stack of hardware. With the expansion of system scale and the improvement of performance, E-class computing has encountered many severe challenges in cutting-edge technology. The brief analysis is as follows: [19-20]

1. Storage access wall. It mainly refers to the mismatch between the processing speed and access and storage speed of the processor, which leads to the serious decline of the computing efficiency of the parallel system. The problem of access and storage wall is still the first problem to improve the computing speed. The appearance of multi-core CPU only improves the computing speed, which not only cannot solve the problem of "memory access wall", but also makes it more and more serious.

2. Communication wall. With the increasing scale of supercomputer system, the impact of interconnection network on computing performance is increasing. The requirement of interconnection network under E-class computing is very high. The problem of "communication wall" has become one of the key factors restricting the innovation and development of supercomputers. The reason why Chinese scientists attach importance to the interconnection network technology independently developed by the Chinese people is also due to the high concern and worry about this issue.

3. Reliability wall. Researchers have been trying to improve the mean time between failures (MTBF) of supercomputer systems, but the reliability of supercomputer systems is becoming more and more serious due to the increasing parallelism. According to the statistical analysis of the current fault-tolerant technology, the MTBF of the system will start to be less than the time used to save the global checkpoints at the scale of $\mathrm{P}$ to $\mathrm{E}$. The consequence of this problem is that the system often crashes due to faults, which seriously affects the performance of the machine. The "reliability wall" hinders the further expansion of the system scale.

4. Energy consumption wall. Among all the challenges, the most serious one is energy consumption. The problem of power consumption hinders the development of high-performance computing in many ways: chips operating at high temperature will have higher failure rate, resulting in the decline of system stability; the sudden increase of power consumption increases the cost of refrigeration technology and chip packaging; supercomputers have a huge demand for energy, resulting in a sharp increase in energy consumption year by year. Therefore, the "energy consumption wall" has become an important measure as important as computing performance. In 2007, scientists in the international industry launched the green 500 ranking synchronized with the top 500, becoming the most authoritative evaluation of the energy consumption level of international supercomputers. According to experts' assessment, if the ideal model of China's future E-class system is constructed with current technology, the power consumption will reach nearly 500MW and the power consumption will reach 4 billion $\mathrm{kwh}$, which is equivalent to one third of the power generation of China's Three Gorges reservoir or one fifth of the annual power consumption of a medium-sized city.

Scientists in the international industry launched the green 500 ranking in 2007, which is synchronized with the top 500, and has become the most authoritative evaluation of the energy consumption level of supercomputers in the world. According to experts' assessment, if the ideal model of China's future E-class system is constructed with current technology, the power consumption will reach nearly 500MW and the power consumption will reach 4 billion kwh, which is equivalent to one third of the power generation of China's Three Gorges reservoir or one fifth of the annual power consumption of a medium-sized city.

Facing the bottleneck of E-level computing technical, scientists began to explore a new form of supercomputer. In 2009, British scientists carried out the research on "Nano-Electroniccomponents". The computer made of this component is called "Optical-Computer". It can not only 
bring higher computing speed with higher performance, but also promote the cross integration of computer technology and biological science. In May 2013, the branch of Max Planck Institute of quantum optics in Germany successfully realized the storage of quantum information with a single atom for the first time, that is, to write the quantum state of a single photon into a quantum, and then read it out after 180 microseconds. This latest breakthrough is expected to help scientists design "quantum computers" to carry out large-scale and high-precision "supercomputing" through quantum splitting and quantum patching. In May 2013, the branch of Max Planck Institute of Quantum Optics in Germany successfully realized the storage of quantum information with a single atom for the first time, that is, to write the quantum state of a single photon into a quantum, and then read it out after 180 microseconds. This latest breakthrough is expected to help scientists design "quantum computers" to carry out large-scale and high-precision "supercomputing" through quantum splitting and quantum patching.

A new era of supercomputer innovation and development in the world is coming!

\section{References}

[1] Hu Shou Ren. History of computer technology [M]. Changsha: University of Defense Science and Technology Press, 2016. 214 215.

[2] Bai RuiXue. decisive battle on the pinnacle [M]. Changsha: Hunan Science and Technology Press, 2014.10 12.

[3] S. Kowalik. Supercomputing [M]. World Publishing Corporation. 1990-11.

[4] Si Hongwei. Seymour Cray: The Father of World Supercomputer [J]. History Research. 2019; 7(1): 1-6

[5] Xinhua military. "Tianhe-1" wins the title of supercomputer in the world Supercomputers become a new arena for power $[\mathrm{N}]$. Xinhua News Agency, 2010. 11. 21.

[6] Zhang Jiuchun, Zhang Baichun. Planning Measures of Chinese
Computing Technology and Soviet Aid in the 1950s [J]. Chinese Science and Technology Historical Materials, 2003 (3).

[7] Bureau of Planning, Chinese Academy of Sciences. Archives of the Chinese Academy of Sciences, 53-3-23.

[8] Department of Physics, Mathematics and Chemistry, Chinese Academy of Sciences. Archives of the Chinese Academy of Sciences, 55-15-3.

[9] School History Editorial Committee of the National Defense Science and Technology University. The School History of University of Defense Science and Technology (1953-1993) [M]. Changsha: University of National Defense Science and Technology Press, 1993. 275 276.

[10] The history editorial board of the University of National Defense Science and Technology. Department of Computer Science and Research Institute, National Defense University (1956-1993) [M]. Internal printing.116 117.

[11] Instructions of vice chairman Deng when listening to the report on computer problems. Archives of College of computer science, NUDT, ZYJ-1-1-5.

[12] Ci Yungui. Report on the development of YH-1. Archives of College of computer science, NUDT, KTYH-I001/06.

[13] National appraisal of Galaxy computer. Archives of computer college, NUDT, KTYH-I001/1.

[14] Xue Ren, Wang Jianwen, Si Hongwei. The Way Beyond [N]. PLA newspaper, 2010.11.19.

[15] Bai Ruixue, Li Zhi, Si Hongwei. Supercomputer super missions in China [J]. Watch Newsweek, 2013. 6. 17.

[16] Liu Yidong, Li Genqun. Research on the Development of China's Computer Industry [M]. Jinan: Shandong Education Press. 2006. 115 116.

[17] https://www.top500.org/lists/2020/11/.

[18] IBM Inc. Some Challenges on Roadfrom Petascale to Exascale [R]. 2010. 4.

[19] Yang Xuejun, the development of Parallel Computing through 60 years [J]. Computer Engineering and Science, 2012 (8).

[20] Xuejun Yang. Thoughts on high-performance computing [J]. National Science Review. 2014. 3. 